\title{
Efficient Conversion of D-Glucose to D-Fructose in the Presence of Organogermanium Compounds
}

\author{
(Received December 10, 2015; Accepted March 4, 2016) \\ (J-STAGE Advance Published Date: March 17, 2016) \\ Takae Nagasawa, ${ }^{1, \dagger}$ Katsuyuki Sato, ${ }^{1}$ Yasuhiro Shimada, ${ }^{1,2}$ and Takafumi Kasumi ${ }^{3}$ \\ ${ }^{1}$ Asai Germanium Research Institute Co., Ltd. \\ (3-131 Suzuranoka-cho, Hakodate, Hokkaido 042-0958, Japan) \\ ${ }^{2}$ The United Graduate School of Agricultural Sciences, Iwate University \\ (3-18-8 Ueda, Morioka, Iwate 020-8550, Japan) \\ ${ }^{3}$ Enzymology and Molecular Biology Laboratory, Department of Chemistry and Life Science, Nihon University \\ (1866 Kameino, Fujisawa, Kanagawa 252-0880, Japan)
}

\begin{abstract}
D-Glucose and D-fructose are isomers of commonly consumed monosaccharides. The ratio of conversion of D-glucose to D-fructose by glucose isomerase (xylose isomerase) is not more than $\mathbf{5 0} \%$. However, addition of an equimolar ratio of the organogermanium compound poly-trans-[(2-carboxyethyl) germasesquioxane] (Ge-132) or its derivative increases the conversion ratio to $80 \%$. In contrast, use of the Lobry de Bruyn-Alberda van Ekenstein transformation with heating results in a lower conversion ratio, less than $30 \%$, whereas addition of an equimolar concentration of Ge-132 or its derivative to this reaction mixture increases the ratio to $73 \%$. Therefore, in this study, we aimed to further analyze the affinity between organogermanium compounds (i.e., Ge-132 and its derivatives) and sugar using ${ }^{1} \mathrm{H}$-nuclear magnetic resonance (NMR) spectrometry. For the dimethyl derivative of Ge-132, the complex formation ratios at $0.25 \mathrm{M}$ (mixing ratio 1:1) were 19 and $74 \%$ for D-glucose and D-fructose, respectively. Additionally, the complex formation constants between monosaccharides and Ge-132 were 1.2 and $46 \mathrm{M}^{-1}$ for D-glucose and D-fructose, respectively. The complex formation capacity was approximately 40 -fold higher for D-fructose than for D-glucose. Therefore, we concluded that the high affinity for the product of isomerization may promote isomerization, and that promotion of sugar isomerization using organogermanium compounds is an effective method for conversion of D-glucose to D-fructose.
\end{abstract}

Key words: organogermanium compound, poly-trans-[(2-carboxyethyl)germasesquioxane], glucose isomerase, alkaline isomerization, cis-diol structure, complex formation constant

\section{INTRODUCTION}

The isomerized sugar known as high fructose syrup is a mixture sugar that is manufactured by conversion of half of the content of D-glucose into D-fructose using an enzymatic reaction catalyzed by glucose isomerase. Recently, D-fructose produced by this isomerization reaction has been used not only in sweeteners but also as a promising chemical intermediate from biomass. ${ }^{1)}$ Therefore, efficient conversion reactions for isomerized sugars are being investigated as a method for increasing the synthesis of such products.

During the production of sweeteners, isomerization is generally stopped after conversion of approximately $42 \%$ of the original substrate, to prevent changes in color and breakdown of the components. The reaction solution is then passed through an ion exchange resin, and the fructose content is concentrated to approximately $90 \%$. This concentrated mixture is then combined with D-glucose, normally yielding a product with $55 \%$ isomerized sugar content. Therefore, improvement in the conversion ratio during the isomerization reaction is critical for the production of

${ }^{\dagger}$ Corresponding author (Tel. +81-138-32-0032, Fax. +81-138-31-0132, E-mail: takaenagasawa@asai-ge.co.jp). isomerized sugar.

The reaction equivalent constant of glucose isomerase is approximately 1.0 (isomerization ratio of $50 \%$ ) at $60{ }^{\circ} \mathrm{C}$, but it increases to 1.348 (isomerization ratio of $57.4 \%$ ) at 100 ${ }^{\circ} \mathrm{C} .{ }^{2)}$ The polarity of the reaction solvent also affects the isomerization ratio; for example, reaction in $85 \%$ ethanol $(\mathrm{v} / \mathrm{v})$ yields an isomerization ratio of $55.9 \%{ }^{3}{ }^{3}$ However, isomerization at $100{ }^{\circ} \mathrm{C}$ is not feasible because of inactivation of the enzyme, and reaction in ethanol-containing solutions proceeds only with 6-12\% D-glucose and at a temperature of $30{ }^{\circ} \mathrm{C}$. Therefore, neither of these modifications to the standard reaction conditions is effective. Some researchers have attempted to shift the equivalence to D-fructose by using reagents with different affinities for the isomers. For example, sodium borate and germanate (i.e., germanium dioxide) can be used as affinity reagents after solubilization under alkaline conditions. Indeed, addition of borate has been reported to increase the isomerization ratio to $88 \%,{ }^{4)}$ and addition of germanate has been reported to increase the isomerization ratio to $90 \% .{ }^{5)}$ However both of these compounds are associated with toxicity; thus, their use is limited. ${ }^{6}$

The water-soluble organogermanium compound poly-trans[(2-carboxyethyl)germasesquioxane], commonly called 
Ge-132,7) is a polymer with the rational formula $\left[\left(\mathrm{GeCH}_{2} \mathrm{CH}_{2} \mathrm{COOH}\right)_{2} \mathrm{O}_{3}\right] \mathrm{n}$, which remains stable under various chemical conditions. The polymer is hydrolyzed into the 3-(trihydroxygermyl)propanoic acid (THGP) monomer in aqueous solution. Ge-132 has been reported to be involved in immune regulation, ${ }^{7)}$ suppression of osteoporosis, ${ }^{8)}$ induction of antioxidative effects, ${ }^{9)}$ antinociceptive effects, ${ }^{10)}$ anti-inflammatory effects, ${ }^{11)}$ antirheumatoid effects, ${ }^{12)}$ and promotion of protoporphyrin IX synthesis. ${ }^{13)}$ Moreover, Ge-132 has been shown to be safe in a variety of toxicity studies. ${ }^{14-22)} \mathrm{Ge}-132$ has recently been used as a component of functional foods and cosmetics. We previously described that THGP is similar to germanium dioxide with respect to the affinity for D-glucose and D-fructose ${ }^{23}$ because of the structural similarity between germanium dioxide and THGP. Phenyl boronic acid has also been shown to have a strong affinity for saccharides, using nuclear magnetic resonance (NMR) analyses. ${ }^{24)}$ However, the differential affinities of THGP and sugar isomers remain unclear.

Therefore, in the present study, we aimed to evaluate the affinity between THGP and sugar isomers using ${ }^{1} \mathrm{H}-\mathrm{NMR}$ analyses. Because of the lack of a germanium nuclear probe for NMR, we used the dimethyl derivate of THGP (DMTHGP) as a reporter molecule and examined the effects of addition of organogermanium compounds of THGP and its derivatives on the enzymatic isomerization reaction and the alkaline isomerization reaction (Lobry de Bruyn-Alberda van Ekenstein transformation). Additionally, we evaluated the affinity between THGP (and its derivatives) and sugar isomers, and proposed a scheme to explain the effects of these compounds on isomerization. To the best of our knowledge, this is the first report describing the affinity between germanium compounds and sugars, as evaluated using ${ }^{1} \mathrm{H}-\mathrm{NMR}$ analyses.

\section{MATERIALS AND METHODS}

Reagents. Figure 1 shows the structures of the six organogermanium compounds (THGP, GPhe, $\mathrm{N}$-Ac-GPhe, GAla, $N$-Ac-GAla, and GSuc) used in this study and the structure of the ${ }^{1} \mathrm{H}-\mathrm{NMR}$ reporter molecule, 3,3-dimethyl3-(trihydroxygermyl)propanoic acid (DM-THGP). All organogermanium compounds used in this study were synthesized at Asai Germanium Research Institute Co., Ltd. D-Glucose (anhydrous) and deuterium oxide (99.9\% deuteration ratio) were purchased from the Merck KGaA (Damstat, Germany). D-Fructose, magnesium sulfate heptahydrate (guaranteed reagent grade), perchloric acid (guaranteed reagent grade), and sodium hydroxide (guaranteed reagent grade) were purchased from Kanto Chemical Co., Ltd. (Tokyo, Japan). The glucose isomerase (D-xylose isomerase EC 5.3.1.5) used was Nagase Enzymes G1-L (Nagase ChemteX Co., Ltd., Osaka, Japan). 3-(N-Morpholino) propanesulfonic acid (MOPS) was purchased from Tokyo Kasei Co., Ltd. (Tokyo, Japan). Distilled water was prepared using Advantec fully automatic distilled water manufacturing equipment (GSR-200; Advantec Toyo Kaisha, Ltd., Tokyo, Japan).

Enzymatic isomerization reaction. The substrate solution was prepared by adding $20 \mathrm{mmol}$ of D-glucose and $0.4 \mathrm{mmol}$ of $\mathrm{MgSO}_{4} \cdot 7 \mathrm{H}_{2} \mathrm{O}$ to $10 \mathrm{~mL}$ of $0.2 \mathrm{M}$ MOPS buffer ( $\left.\mathrm{pH} 8\right)$.

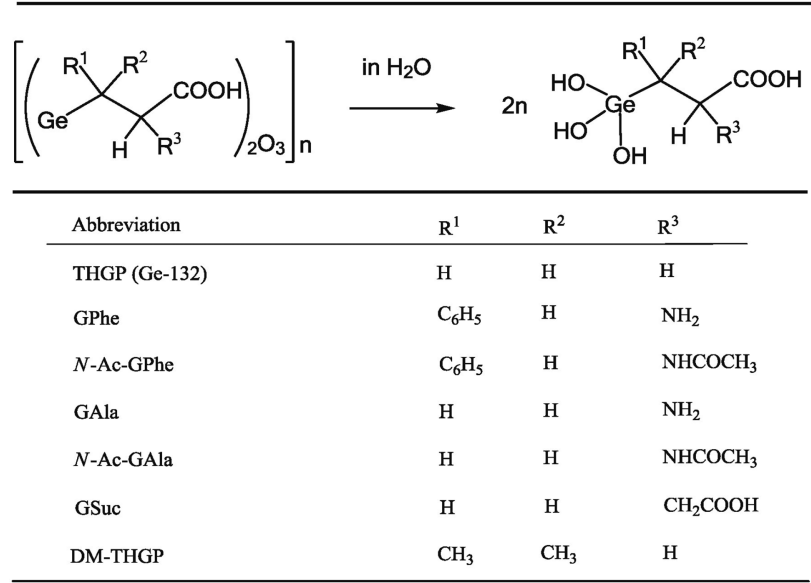

Fig. 1. Organogermanium compounds used in the experiments.

The structures of the compounds in aqueous solution and their abbreviations are shown.

Glucose isomerase $(5.69 \mathrm{mg} / \mathrm{mL}$ Nagase Enzymes G1-L) was filtered through a membrane filter $(0.22 \mu \mathrm{m}$; Millex-GS, Merck Millipore, Billerica, USA). To prepare each organogermanium solution, the organogermanium compound was dissolved to a concentration of $2 \mathrm{M}$ (as the molar concentration of the germanium monomer compound), with adjustment to $\mathrm{pH} 8$ using sodium hydroxide solution. The reaction mixture was prepared by adding $0.8 \mathrm{~mL}$ of the $2 \mathrm{M}$ organogermanium solution to $0.8 \mathrm{~mL}$ of the $2 \mathrm{M}$ substrate solution as described above. Subsequently, $16 \mu \mathrm{L}$ of the enzyme solution was added to the reaction mixture. The final reaction mixtures (pH 7.8) contained $1 \mathrm{M}$ D-glucose and $1 \mathrm{M}$ organogermanium. The control solution was prepared using distilled water rather than the organogermanium solution. All preparation steps were performed under ice-cold conditions. Reaction mixtures $\left(0.1 \mathrm{~mL}\right.$ each) were incubated at $60{ }^{\circ} \mathrm{C}$ with shaking $(120 \mathrm{rpm})$ for $15,30,45,60,90,120,150,180,210$, or $240 \mathrm{~min}$. The reaction mixture was then placed quickly on ice water, and the reaction was stopped by adding $0.1 \mathrm{~mL}$ of $0.5 \mathrm{M}$ perchloric acid solution.

Alkaline isomerization reaction. To prepare each organogermanium solution, the organogermanium compound was dissolved to a concentration of $2 \mathrm{M}$ (as the molar concentration of the germanium monomer compound), with adjustment to $\mathrm{pH} 12$ using sodium hydroxide solution. The reaction mixture was prepared by adding $2.5 \mathrm{~mL}$ of the organogermanium solution to $2 \mathrm{~mL}$ of $2.5 \mathrm{M}$ D-glucose solution, and the $\mathrm{pH}$ was adjusted to 12 using $0.2-0.4 \mathrm{~mL}$ of $10 \mathrm{M}$ sodium hydroxide solution. The final concentrations of D-glucose and organogermanium compound in the reaction mixture were $1 \mathrm{M}$. The control solution was prepared using distilled water rather than the organogermanium solution. All preparation steps were performed under ice-cold conditions. Reaction mixtures $(0.5 \mathrm{~mL}$ each $)$ were incubated at $60{ }^{\circ} \mathrm{C}$ with shaking (120 rpm) for 5, 10, 15, 20, 25, 30, 40, 50, or $60 \mathrm{~min}$, and then quickly placed on iced water.

Analysis of D-glucose and D-fructose using high-performance liquid chromatography (HPLC). The reaction mixtures were diluted 180-200-fold with distilled water and then centrifuged for $3 \mathrm{~min}$ at $13,500 \times \mathrm{G}$ at $1{ }^{\circ} \mathrm{C}$. D-Glucose and $\mathrm{D}$-fructose contents in the supernatants were determined by HPLC. The sugar isomers were separated with a Shim-pack SCR-101C SCR (C) column $(7.9 \phi \times 300 \mathrm{~mm}$, 
$4 \phi \times 50 \mathrm{~mm}$; Shimadzu Corporation, Kyoto, Japan) at $80{ }^{\circ} \mathrm{C}$. The mobile phase was distilled water, introduced at a flow rate of $1.0 \mathrm{~mL} / \mathrm{min}$. The sample volume applied was 10 $\mu \mathrm{L}$. An LC-10A instrument, equipped with an RID-6A refractive index detector (Shimadzu), was used for detection. NMR measurement. NMR spectra were recorded at $20 \pm$ $0.5^{\circ} \mathrm{C}$ using a Varian Gemini 300 spectrometer (Agilent Technologies, Santa Clara, USA) equipped with a 5-mm diameter $\mathrm{C} / \mathrm{H}$ dual probe. Chemical shifts were expressed in ppm relative to the $\mathrm{H}$ resonance of HOD in solvent, which was offset by $4.80 \mathrm{ppm}$.

Comparison of the affinity of THGP and its derivatives for D-glucose and D-fructose. To elucidate the mechanism through which THGP derivatives promote isomerization, we measured the complex formation constants $\left(K_{\mathrm{s}}\right)$ for D-glucose and D-fructose using DM-THGP. First, the complex formation ratios for D-glucose and D-fructose in the presence of DM-THGP were measured using ${ }^{1} \mathrm{H}-\mathrm{NMR}$ under the following conditions: sugar concentration of $0.25 \mathrm{M}$ constant; DM-THGP concentrations of $0.075,0.10$, $0.125,0.15,0.175,0.20,0.225,0.25,0.275$, or $0.30 \mathrm{M}$; and $\mathrm{pH}$ range of 6.0 to 7.4. Subsequently, the ${ }^{1} \mathrm{H}-\mathrm{NMR}$ integral values of the sugar $(0.25 \mathrm{M}$ constant concentration) were used to correct the concentration of DM-THGP. Complexation ratios were calculated using the following equation: $C_{\mathrm{r}}$ $=I_{\mathrm{c}} /\left(I_{\mathrm{c}}+I_{\mathrm{f}}\right)$, where $C \mathrm{r}, I_{\mathrm{c}}$, and $I_{\mathrm{f}}$ are the complexation ratio, integral value of the methyl proton signal corresponding to the DM-THGP-sugar complex, and integral value of the methyl proton signal corresponding to DM-THGP, respectively. Then, using the complexation ratios from the formula above, the $K_{\mathrm{s}}$ values of DM-THGP for D-glucose and D-fructose were calculated from the slope of the complex concentration/free sugar concentration ratio plotted against the free DM-THGP concentration based on the equation $K_{\mathrm{s}}$ $=[\mathrm{ML}] /[\mathrm{M}][\mathrm{L}]\left(\right.$ where $K_{\mathrm{s}},[\mathrm{ML}],[\mathrm{M}]$, and $[\mathrm{L}]$ are the complex formation constants, molar concentration of the sugar-DM-THGP complex, molar concentration of free DM-THGP, and molar concentration of the free sugar, respectively).

\section{RESULTS}

\section{Effects of organogermanium compounds on enzymatic} isomerization.

Figure 2 shows the results of the enzymatic isomerization of D-glucose to D-fructose in the presence of organogermanium compounds. Approximately $50 \%$ of D-glucose was isomerized into $\mathrm{D}$-fructose under normal reaction conditions (control). In contrast, more than $70 \%$ (74-85\%) of D-glucose was converted to D-fructose when equimolar concentrations of organogermanium compounds were added. The conversion of D-glucose to D-fructose was increased to $80 \%$ (120 min, over $30 \%$ higher than control) following addition of THGP. Moreover, addition of GSuc, a carboxymethyl-substituted derivative, resulted in the greatest increase in the conversion rate, up to $85 \%$ (150 min). $N$-Ac-GPhe and $N$-Ac-GAla, which contained an amino group protected with an acetyl group, increased the conversion rates to $84 \%$ (150 $\mathrm{min}$ ) and $81 \%$ (150 $\mathrm{min})$, respectively. Moreover, addition of these organogermanium compounds to the enzyme isomerization reaction system at equimolar concentrations increased the conversion ratios to up to 1.7 times that of the control.

\section{Effects of the organogermanium compounds on alkaline isomerization.}

Figure $3 \mathrm{~A}$ shows the results of alkaline isomerization from D-glucose to D-fructose under alkaline conditions in the presence of organogermanium compounds. Under normal reactions conditions (control, $15 \mathrm{~min}$ ), a D-fructose yield of approximately $30 \%$ was obtained. In contrast, a D-fructose yield of more than $70 \%$ was obtained when an equimolar concentration of organogermanium was added to the reaction mixture. For example, the fructose yield increased to $73 \%$ (30 $\mathrm{min}$ ) and $70 \%$ (40 min) after addition of THGP (Ge-132) or $N$-Ac-GAla, respectively. The yield of $\mathrm{D}$-fructose from alkaline isomerization of D-glucose was highest after addition of THGP (Ge-132). The remaining ratio of D-glucose and the remaining ratio of total saccharides at each reaction time point are shown in Fig. 3B and

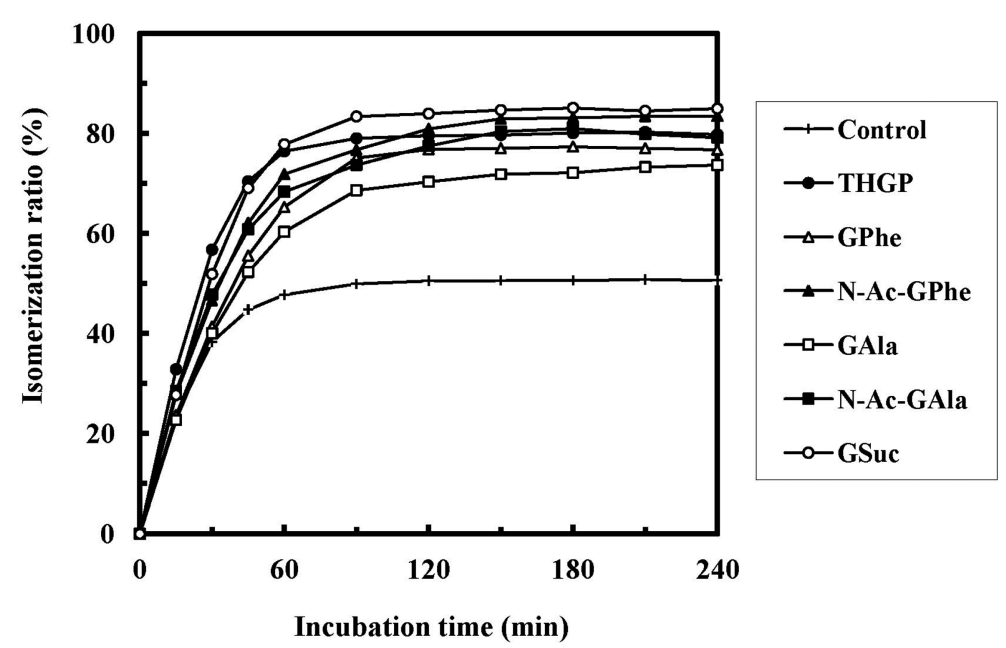

Fig. 2. Time course of enzymatic isomerization ratios in the presence of organogermanium compounds.

Enzymatic isomerization from D-glucose to D-fructose using a glucose isomerase in the presence of equimolar concentrations of organogermanium compound (final concentration, $1 \mathrm{M}$; reaction temperature, $60{ }^{\circ} \mathrm{C} ; \mathrm{pH} 7.8$ ). The concentration of each sugar was determined by HPLC. Isomerization ratio $(\%)=\mathrm{D}$-fructose concentration at each reaction time/initial D-glucose concentration $\times 100$. 
A

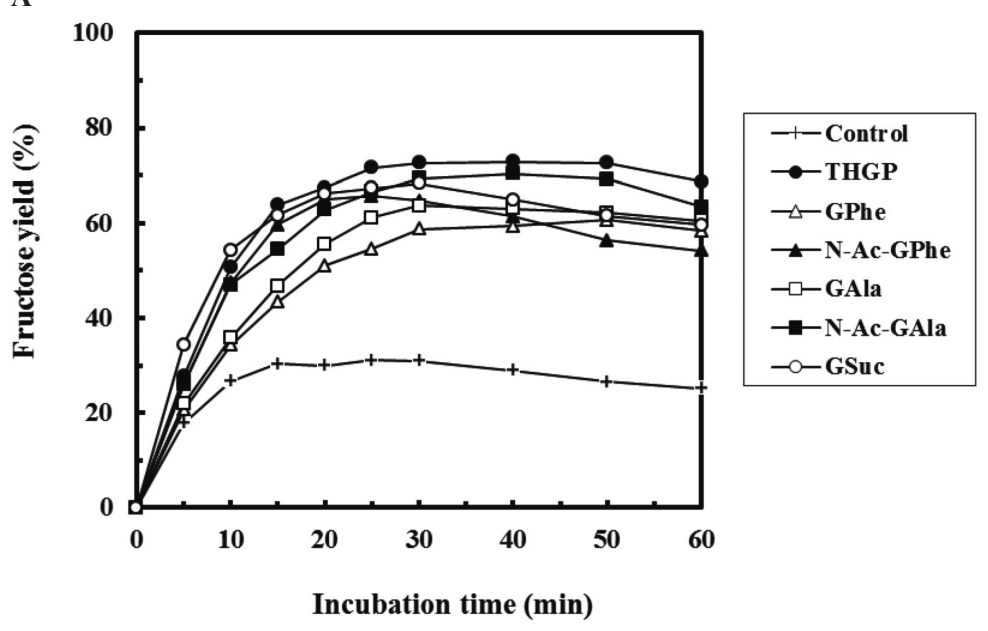

B

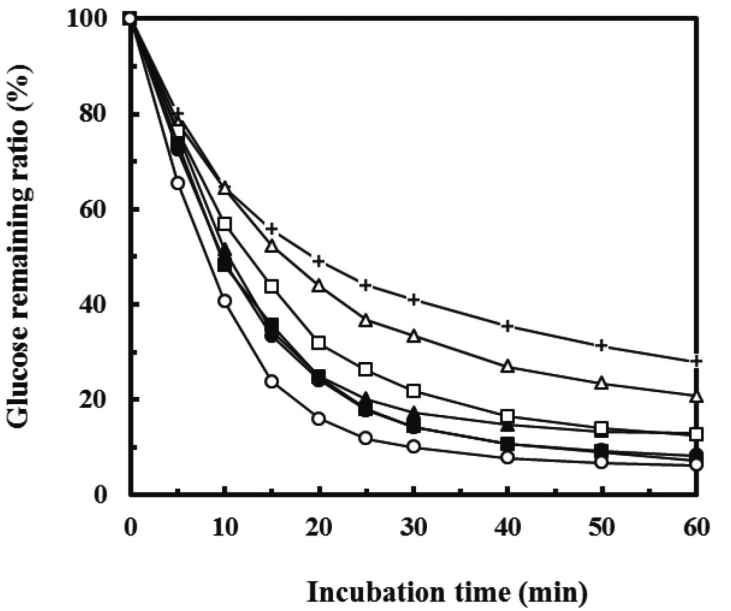

$-1-$ Control

$\rightarrow$ THGP

$\neg-$ GPhe

$\leftarrow$ N-Ac-GPhe

$\rightarrow-$ GAla

$\rightarrow$-N-Ac-GAla

$\rightarrow$ GSuc

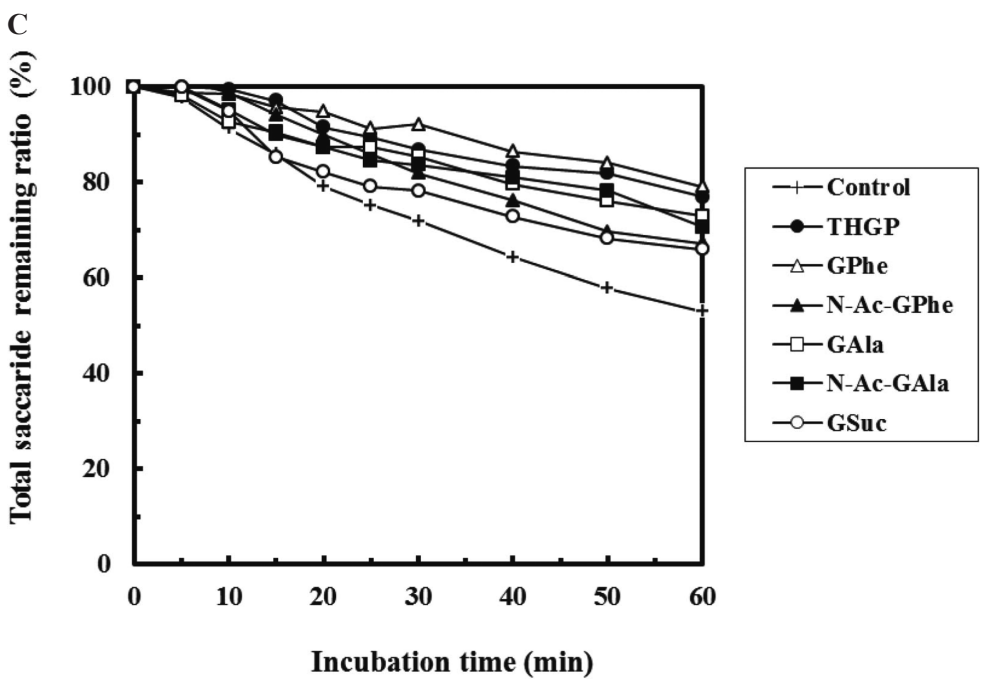

Fig. 3. Effects of organogermanium compounds on alkaline isomerization from D-glucose to D-fructose.

A, D-Fructose yield $=\mathrm{D}$-fructose concentration at each reaction time/initial Dglucose concentration $\times 100$; $\mathrm{B}$, D-glucose remaining ratio $=\mathrm{D}$-glucose concentration at each reaction time/initial D-glucose concentration $\times 100$; C, Total saccharide remaining ratio $=\mathrm{D}$-glucose remaining ratio $+\mathrm{D}$-fructose yield at each reaction time. The data were obtained by HPLC analysis of saccharides. A Shimpack HPLC column was used at $80{ }^{\circ} \mathrm{C}$. Distilled water was used as the mobile phase, and the flow rate was $1.0 \mathrm{~mL} / \mathrm{min}$. A refractive index (RI) detector was used for detection. 
$3 \mathrm{C}$, respectively. The total amount of saccharide decreased under alkaline conditions. The highest ratio of remaining D-glucose was observed in the control group after reaction for $60 \mathrm{~min}(28 \%)$; under these conditions, the ratio of remaining total saccharides was lowest $(53 \%)$. The $\mathrm{pH}$ value was decreased by the organic acids or some other compounds produced as a by-product of the reaction (data not shown). The most dramatic drop in $\mathrm{pH}$ was observed for the control group. In contrast, addition of organogermanium compounds suppressed this reduction in $\mathrm{pH}$. Finally, addition of equimolar concentrations of organogermanium compounds to the alkaline isomerization reaction increased the D-fructose yield up to 2.4-fold that of the control.

Comparison of the complex formation constants (Ks) for the sugar-THGP structure using a ${ }^{1} H-N M R$ reporter molecule.

The $K_{\mathrm{s}}$ values of THGP derivatives with sugar were estimated using DM-THGP as a reporter molecule in ${ }^{1} \mathrm{H}-\mathrm{NMR}$ analyses. The proton NMR spectrum of DM-THGP gave only two singlet signals corresponding to the methyl and methylene groups, which do not exhibit proton coupling (Fig. 4A). However, signals corresponding to the complex
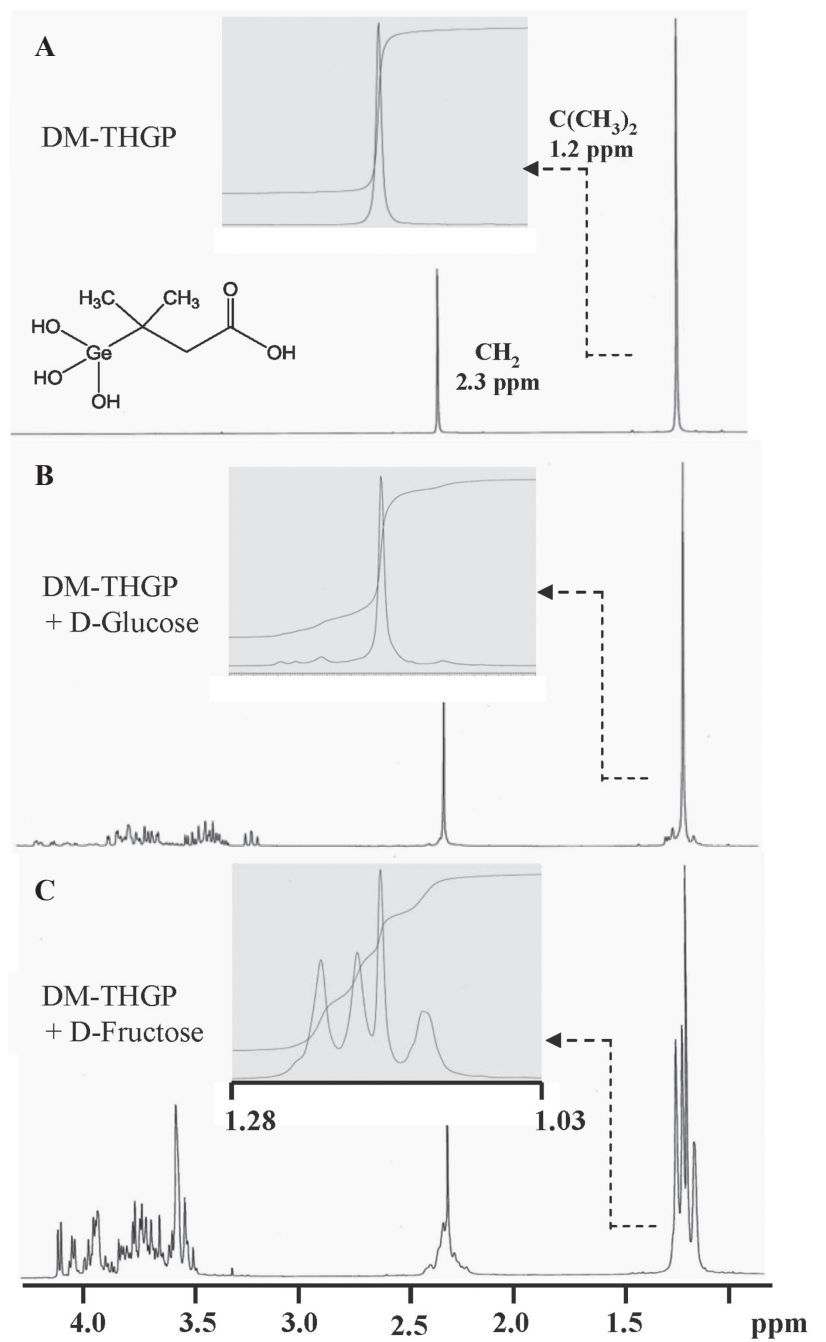

Fig. 4. ${ }^{1} \mathrm{H}-\mathrm{NMR}$ spectra $(300 \mathrm{MHz})$ of methyl and methylene signals of DM-THGP and its complexes with sugars in the $0.8-4.30$ ppm region.

The spread spectra of the methyl signals in the $1.03-1.28 \mathrm{ppm}$ region are also shown. A, DM-THGP; B, with D-glucose, 1:1, 0.25 M; C, with D-fructose, $1: 1,0.25 \mathrm{M}$, at $20^{\circ} \mathrm{C}$ in $\mathrm{D}_{2} \mathrm{O}$, pH 6.0-7.4. between DM-THGP and sugar were observed near the original signals as multiple shifting signals (Figs. 4B and 4C). The complex formation ratios were calculated using the integral value ratios. Therefore, DM-THGP could be used as a ${ }^{1} \mathrm{H}-\mathrm{NMR}$ reporter molecule for examining the interaction of the THGP structure with carbohydrates. The $K_{\mathrm{s}}$ values of DM-THGP for D-glucose and D-fructose were calculated as described in the Materials and Methods, and plots of the complex concentration and free sugar concentration versus the free-DM-THGP concentration are shown in Fig. 5. The mean $K_{\mathrm{s}}$ values were $K_{\mathrm{s}}{ }^{\text {(glucose) }}=1.2 \mathrm{M}^{-1}$ and $K_{\mathrm{s}}{ }^{\text {(fructose) }}=$ $46 \mathrm{M}^{-1}$. Thus, the affinity of DM-THGP for D-fructose was approximately 40 times higher than that for D-glucose.

\section{DISCUSSION}

As described above, organogermanium compounds with a THGP structure promoted the conversion from D-glucose to D-fructose in both the enzymatic reaction and the alkaline isomerization reaction. The high affinity of the THGP structure for D-fructose was revealed using the ${ }^{1} \mathrm{H}-\mathrm{NMR}$ reporter molecule DM-THGP. Because THGP derivatives bound more strongly to D-fructose than to D-glucose, the equilibrium of the isomerization reaction would be expected to be shifted toward D-fructose. This mechanism could explain the observed effects of THGP derivatives on enzymatic isomerization from D-glucose to D-fructose. Figure 6 shows the complex formation reaction scheme between a saccharide and THGP derivative. A similar mechanism has also been reported for the enzyme isomerization induced by the boron compound. ${ }^{25)}$ In contrast, the association constants of benzoxaborole, which can form complexes with cis-diol at neutral $\mathrm{pH}$, have been reported to be $664 \mathrm{M}^{-1}$ for $\mathrm{D}$-fructose and $21 \mathrm{M}^{-1}$ for D-glucose. ${ }^{26)}$ These values are approximately 15 times higher than those of DM-THGP obtained in the present study. This difference in affinity may reflect the relative size of boron and germanium atoms. The reduced affinity of the interaction may be preferred from the viewpoint of food safety of THGP (Ge-132).

THGP derivatives also promoted alkaline isomerization from D-glucose to D-fructose. The reaction for alkaline isomerization from D-glucose to D-fructose proceeded via the 1,2-enediol intermediate. ${ }^{1,27)}$ Additionally, high complex formation capacity of the cis-diol group of the catechol of THGP, including a penta-coordinate Ge, has been reported. ${ }^{28)}$ Thus, stabilization of the 1,2-enediol intermediate by complex formation with the THGP derivative is thought to occur, which would favor for the formation of D-fructose to stabilize the transition state. In addition, part of the D-fructose molecule will have a negative charge because of complex formation with the Ge anion of the THGP derivative, as shown in Fig. 6D. This negative charge will help to protect the D-fructose molecule from the attack of the alkaline reagent, because negative charges would repel each other. In fact, the ratio of remaining total saccharides in the presence of THGP derivatives was higher than in the control.

THGP derivatives promoted enzyme isomerization (150 $\min$ ) in the following order: GSuc $\approx N$-Ac-GPhe $>N$-Ac-GAla $\approx$ THGP $>$ GPhe $>$ GAla $>$ control. GSuc and $N$-Ac-GPhe, which possess structures favoring cyclization of THGP 


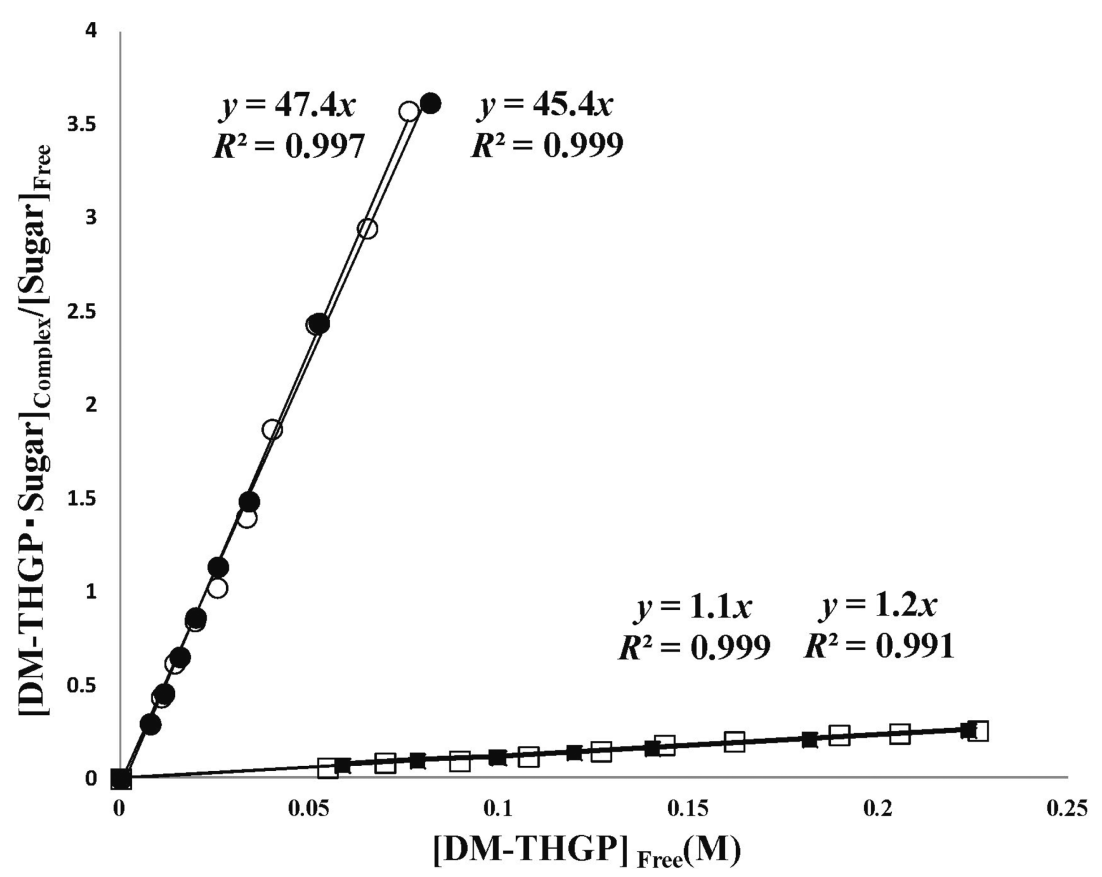

Fig. 5. Comparison of the complex formation constants $(K \mathrm{~s})$ of DM-THGP versus Dglucose $(\square$ and $\square$ ) and D-fructose $(\bigcirc$ and $\bigcirc)$.

Plots of the complex concentration and free sugar concentration versus the free DMTHGP concentration at $20^{\circ} \mathrm{C}$ in $\mathrm{D}_{2} \mathrm{O}, \mathrm{pH} 6.0^{-7.4}$.

$\mathbf{A}$

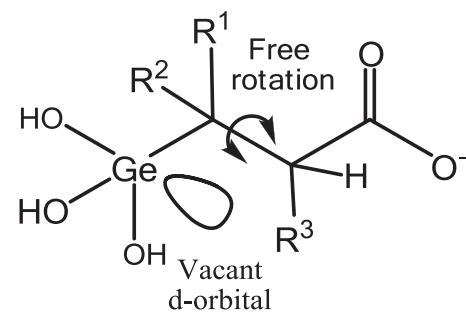

B<smiles></smiles>

at around neutral $\mathrm{pH}$

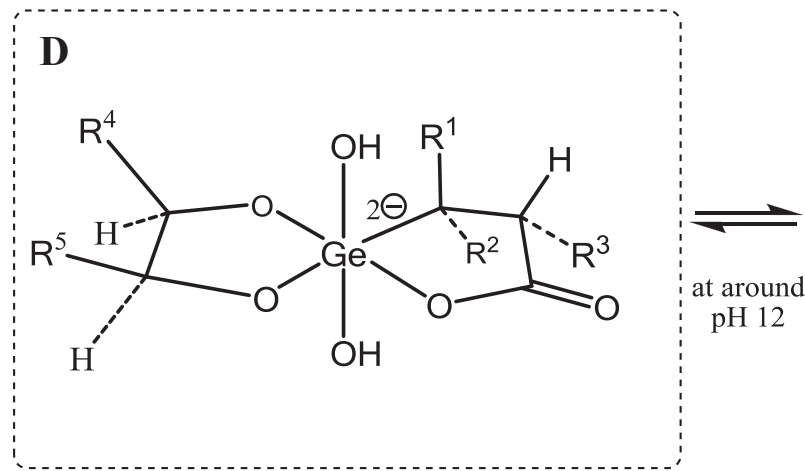<smiles>[R7]C(O)[C@H]([R7])[2H]</smiles>

C

cis-diol compound<smiles>[R]C1([R])O[Ge](O)(O)OC(=O)C1([R])[R]</smiles>

Fig. 6. Scheme of complex formation reaction between THGP structures and cis-diol compounds.

Carboxylate anion of the THGP structure including free rotation of the propanoic acid side chain bond to the vacant $\mathrm{d}$-orbital of the Ge atom (A) in the intermolecular structure (B). The resulting pentacoordinate trihydroxygermanium form reacted with cis-diol compounds, including a dehydration step, and then formed stable complexes at approximately neutral $\mathrm{pH}$ (C). The generation of the hexa-coordinate germanium complex was estimated to occur at approximately $\mathrm{pH} 12$ (D).

derivatives (i.e., lactone-like ring formation of penta-coordinate germanium) by steric factors, showed relatively higher isomerization-promoting effects than the other derivatives, supporting the mechanism described above. A similar order was also observed in the initial alkaline isomerization reaction, supporting the strength of complex formation between THGP derivatives and the 1,2-enediol intermediate. However, GSuc and $N$-Ac-GPhe, which have sterically hindered structures, showed less protective effects on D-fructose from alkaline decomposition (D-fructose yields at $25 \mathrm{~min}$ and later were decreased) than THGP (Ge-132) and $N$-Ac-GAla, which have simple structures. The hepta- 
coordinate Ge structure of THGP at $\mathrm{pH} 12$ or more was estimated (data not shown), and the potential protective effects of this structure on D-fructose are believed to be related to the steric stability of hepta-coordinate Ge complexes, as shown in Fig. 6D.

In summary, the results of the present study indicated that promotion of sugar isomerization using organogermanium compounds was an effective method for conversion from D-glucose to D-fructose. However, organogermanium compounds are relatively expensive. Therefore, the development of a recycling system for organogermanium compounds using selective capture materials ${ }^{29)}$ or electrodialysis instruments for organogermanium compounds is necessary for continued advancement in this field.

\section{ACKNOWLEDGEMENTS}

We are very grateful to Dr. Norihiro Kakimoto and Dr. Keiji Umeda for their helpful advice regarding the study design. We also thank Dr. Mitsuo Akiba for assistance with management of the study and Dr. Takashi Nakamura who provided valuable advice. A part of this study was performed at the National Food Research Institute, NARO, Japan; therefore, we thank the researchers who provided assistance with the experiments.

\section{REFERENCES}

1) M. Moliner, Y.R. Leshkov, and M. E. Davis: Tin-containing zeolites are highly active catalysts for the isomerization of glucose in water. Proc. Natl. Acad. Sci. USA, 107, 6164-6168 (2010).

2 ) Y.B. Tewari and R.N. Goldberg: Thermodynamics of the conversion of aqueous glucose to fructose. Appl. Biochem. Biotechnol., 11, 17-24 (1985)

3 ) K. Visuri and A.M. Klibanov: Enzymatic production of high fructose corn syrup (HFCS) containing $55 \%$ fructose in aqueous ethanol. Biotechnol. Bioeng., 30, 917-920 (1987).

4 ) Y. Takasaki: Studies on sugar-isomerizing enzymes. Effect of borate on glucose-fructose isomerization catalyzed by glucose isomerase. Agric. Biol. Chem., 35, 1371-1375 (1971).

5 ) S.A. Barker, H. Pelmore, and P.J. Somers: Effect of oxyanions on the D-glucose isomerase catalysed equilibrium: 2. Effect of germanate on the equilibrium of D-glucose and D-fructose with immobilized D-glucose isomerase. Enzyme Microb. Technol., 5, 121-124 (1983).

6 ) T. Sanai, S. Okuda, K. Onoyama, N. Oochi, Y. Oh, K. Kobayashi, K. Shimamatsu, S. Fujimi, and M. Fujishima: Germanium dioxide-induced nephropathy: a new type of renal disease. Nephron, 54, 53-60 (1990).

7 ) H. Aso, F. Suzuki, T. Yamaguchi, Y. Hayashi, T. Ebina, and N. Ishida: Induction of interferon and activation of NK cells and macrophages in mice by oral administration of Ge-132, an organic germanium compound. Microbiol. Immunol., 29, 65-74 (1985).

8 ) H. Matsumoto, G.Z. Jiang, T. Hashimoto, N. Kuboyama, J. Yamane, K. Nonaka, and A. Fujii: Effect of organic germanium compound (Ge-132) on experimental osteoporosis in rats: the relationship between transverse strength and bone mineral density (BMD) or bone mineral content (BMC). Int. J. Oral-Med. Sci., 1, 10-16 (2002).

9 ) T. Nakamura, T. Nagura, M. Akiba, K. Sato, Y. Tokuji, M. Ohnishi, and K. Osada: Promotive effects of the dietary organic germanium poly-trans-[(2-carboxyethyl)germasesquioxane $](\mathrm{Ge}-132)$ on the secretion and antioxidative activity of bile in rodents. J. Health Sci., 56, 72-80 (2010).

10) Y. Suzuki and K. Taguchi: Pharmacological studies of carboxyethylgermanium sesquioxide (Ge-132) (1). Pharmacometrics, 26, 803-810 (1983). (in Japanese)

11) K. Sasaki, M. Ishikawa, K. Monma, and G. Takayanagi: Effect of carboxyethylgermanium sesquioxide $(\mathrm{Ge}-132)$ on the acute inflammation and $\mathrm{CCl}_{4}$-induced hepatic damage in mice. Pharma- cometrics, 27, 1119-1131 (1984). (in Japanese)

12) L. Pronai and S. Arimori: Decreased plasma superoxide scavenging activity in immunological disorders carboxyethylgermanium sesquioxide (Ge-132) as a promoter of prednisolone. Biotherapy, 4, 1-8 (1992).

13) T. Nakamura, M. Saito, Y. Shimada, H. Fukaya, Y. Shida, and Y. Tokuji: Induction of aminolevulinic acid synthase gene expression and enhancement of metabolite, protoporphyrin IX, excretion by organic germanium. Eur. J. Pharmacol., 653, 75-81 (2011).

14) T. Nagata, T. Nagata, Y. Aramaki, M. Enomoto, H. Isaka, and J. Otuka: Chronic intravenously toxicity study with carboxyethylgermanium sesquioxide in beagle-dogs. Pharmacometrics, 16, 613-636 (1978). (in Japanese)

15) H. Nagai, K. Hasegawa, and K. Shimpo: Reproductive study of rats intraperitoneally with carboxyethylgermanium sesquioxide (Ge-132). Pharmacometrics, 20, 271-280 (1980). (in Japanese)

16) S. Nakayama, T. Tsuji, and K. Usami: Acute toxicity study of organic germanium (Ge-132) in mice and rats. Syowa Igakukai Zasshi, 46, 227-235 (1986). (in Japanese)

17) Y. Sugiya, S. Sakamaki, T. Sugita, Y. Abo, and H. Satoh: Subacute oral toxicity of carboxyethylgermanium sesquioxide (Ge-132) in rats. Pharmacometrics, 31, 1181-1190 (1986). (in Japanese)

18) Y. Sugiya, T. Sugita, S. Sakamaki, Y. Abo, and H. Satoh: Subacute and chronic intraperitoneal toxicity of carboxyethylgermanium sesquioxide (Ge-132) in rats. Pharmacometrics, 32, 93-111 (1986). (in Japanese)

19) Y. Sugiya, K. Eda, K. Yoshida, S. Sakamaki, and H. Satoh: Reproductive and teratogenic studies of carboxyethylgermanium sesquioxide (Ge-132) (1), fertility study in rats by intravenous administration. Pharmacometrics, 32, 113-121 (1986). (in Japanese)

20) Y. Sugiya, K. Yoshida, S. Sakamaki, K. Eda, and H. Satoh: Reproductive and teratogenic studies of carboxyethylgermanium sesquioxide (Ge-132) (2), teratogenesis study in rats by intravenous administration. Pharmacometrics, 32, 123-138 (1986). (in Japanese)

21) Y. Sugiya, K. Yoshida, K. Eda, S. Sakamaki, and H. Satoh: Reproductive and teratogenic studies of carboxyethylgermanium sesquioxide (Ge-132) (3), perinatal and postnatal studies in rats by intravenous administration. Pharmacometrics, 32, 139-152 (1986). (in Japanese)

22) T. Sanai, S. Okuda, K. Onoyama, N. Oochi, S. Takaichi, V. Mizuhira, and M. Fujishima: Chronic tubulointerstitial changes induced by germanium dioxide in comparison with carboxyethylgermanium sesquioxide. Kidney Int., 40, 882-890 (1991).

23) Y. Shimada, K. Sato, Y. Tokuji, and T. Nakamura: Nuclear magnetic resonance studies of the interactions between the organic germanium compound Ge-132 and saccharides. Carbohydr. Res., 407, 10-15 (2015).

24) K. Oshima, H. Toi, and Y. Aoyama: Complexation of phenylboronic acid with alkylglycopyranosides and related polyols as studied by ${ }^{11}$ B NMR spectroscopy. Carbohydr. Lett., 1, 223-230 (1994).

25) N.-H. Kim, H.-J. Kim, D.-I. Kang, K.-W. Jeong, J.-K. Lee, Y. Kim, and D.-K. Oh: Conversion shift of D-fructose to D-psicicose for enzyme-catalyzed epimerization by addition of borate. Appl. Environ. Microbiol., 74, 3008-3013 (2008).

26) A. Mahalingam, A.R. Geonnotti, J. Balazarini, and P.F. Kiser: Activity and safety of synthetic lectins based on benzoboroxolefunctionalized polymers for inhibition of HIV entry. Mol. Pharm., 8, 2465-2475 (2011).

27) R.W. Nagorski and J.P. Richard: Mechanistic imperatives for aldose-ketose isomerization in water: specific, general base- and metal ion-catalyzed isomerization of glyceraldehyde with proton and hydride transfer. J. Am. Chem. Soc., 123, 794-802 (2001).

28) T. Nakamura, Y. Shimada, T. Takeda, K. Sato, M. Akiba, and H. Fukaya: Ornanogermanium compound, Ge-132, forms complexes with adrenaline, ATP and other physiological cis-diol compounds. Future Med. Chem., 7, 1233-1246 (2015).

29) T. Mochizuki, K. Saito, K. Sato, M. Akiba, and T. Sugo: Recovery of p.t-CEtGeO using chelating porous membranes prepared with various compositions of dioxane/water solvent. J. Ion Exchange, 18, 68-74 (2007). 